\title{
Finding Pulsar Emission Heights from Dual-Frequency Observations
}

\author{
Leszek A. Nowakowski \\ Department of Physics, University of Puerto Rico, Mayagüez, PR 00681
}

\begin{abstract}
We present a method that allows to find the radial separation of regions emitting individual components of pulsar average profiles at two radio frequencies. It may also be used for single-frequency observations in pulsars that have intensity-dependent average profiles and/or mode-switching. Preliminary results for three radio pulsars are presented, obtained using average profiles from non-simultaneous observations.
\end{abstract}

\section{Introduction}

We assume that: (i) magnetic field structure is purely dipolar, (ii) emission is beamed tangentially to the field lines, (iii) radius-to-frequency mapping (RFM) influences widths of the average profiles and individual (Gaussian) components, (iv) three combined effects: retardation, aberration, and magnetic field curvature cause an apparent change of the arrival times of the samples of the profile at the observers site. When the emitting region moves radially away from the neutron star, combined effects of retardation, aberration, and geometry of the magnetic field lines cause an apparent change of the arrival times of the samples of the profile at the observer's site according to the equation (Nowakowski $1999 \mathrm{a}, \mathrm{b})$ :

$$
\Phi_{U}=\Phi_{L}-\left(\frac{1+\sin \Theta}{c}\right) r-\frac{3\left(\Phi_{0}-\Phi_{L}\right) r^{\frac{1}{2}}}{2\left(\Phi_{0}-\Phi_{b}\right) \sin \Theta}\left(\frac{P}{2 \pi c}\right)^{\frac{1}{2}}
$$

where subscripts $\mathrm{U}$ and $\mathrm{L}$ denote samples originating from the upper and lower emission heights, $\Phi_{b}$ is the phase of the beginning of the lower-altitude profile, and $\Theta$ is the angle between the magnetic and rotational axes (Rankin 1990, Lyne \& Manchester 1998). The magnitude of that shift is non-linear and depends on the distance of each sample of the low-altitude profile from the magnetic meridian, $\Phi_{0}$, and on the radial separation of the two emitting regions, $r$. Since the three effects have the same sign in the leading part of the profile but the geometrical term changes sign in the trailing part of the profile, the leading part of the profile expands more with emission height than the trailing part.

In a single average profile it is assumed that all radiation that forms that component comes from the same emission height. In multi-component profiles that assumption may be false. Eq.(1) was developed for the last open magnetic field lines, and the same lines should be taken as $\Phi_{b}$. We have used phases where the intensity of the leading edge of the lower-altitude profile drops to $\approx 10^{-4}$ of the maximum. 


\section{Profiles Emitted from two Emission Heights}

In Eq.(1) we replace everything constant for a given pulsar with $A$ and $B$ :

$$
\Phi_{U}=\Phi_{L}-A r-\left(\Phi_{0}-\Phi_{L}\right) B r^{\frac{1}{2}}
$$

Two profiles at different frequencies may be presented in a way used first by Kardashev et al. (1986, Fig.5), and then interpreted by Gil (1991). The slope of the linear fit to the Kardashev's graph is obtained from Eq.(2):

$$
S \equiv \frac{d \Phi_{L}}{d \Phi_{U}}=\frac{1}{1+B r^{\frac{1}{2}}}
$$

The slope is also equal to the ratio of the widths, $S=\sigma_{L} / \sigma_{U}$, where $\sigma_{L}$ and $\sigma_{U}$ are widths of the lower- and upper-level component of the profile. Combining the above equations, and solving for $r$, results in:

$$
r=2 \pi c \frac{\left(\sigma_{U}-\sigma_{L}\right)^{2} \sin ^{2} \Theta\left(\Phi_{o}-\Phi_{b}\right)^{2}}{2.25 P \sigma_{L}^{2}}
$$

It is easy to show that the same equation applies to profile widths, $W_{U}$ and $W_{L}$.

\section{Preliminary Results}

For PSR 0148-06 and 0149-16 we have used average profiles at 408, 610, and $1418 \mathrm{MHz}$. For PSR $0301+19$ we have used profiles at 610 and $1408 \mathrm{MHz}$. The average profiles were obtained from the European Pulsar Network (Gould \& Lyne 1998). Each average profile was approximated with three and five Gaussian components. Radial separations obtained for individual components, and lower and upper limits obtained from the entire profiles, are as follows: PSR 0148-06 (leading component only): $1418-408 \mathrm{MHz}-16 \mathrm{~km} ; 1418-610 \mathrm{MHz}-3.5 \mathrm{~km}$; $610-408 \mathrm{MHz}-3 \mathrm{~km}$. For the entire profiles: $16-48 \mathrm{~km}, 6-12 \mathrm{~km}$, and $2-18 \mathrm{~km}$, respectively. PSR 0149-16 (leading and trailing component): $1408-408 \mathrm{MHz}-$ $0.2 \mathrm{~km}$ and $11 \mathrm{~km}, 1408-610 \mathrm{MHz}-1.2 \mathrm{~km}$ and $20 \mathrm{~km}$, and $610-408 \mathrm{MHz}-$ $0.06 \mathrm{~km}$ and $0.02 \mathrm{~km}$. For the entire profiles: $2-5 \mathrm{~km}, 2-9 \mathrm{~km}$, and $0.1-1 \mathrm{~km}$, respectively. PSR $0301+19$ (leading component): $1408-610 \mathrm{MHz}-1.5 \mathrm{~km}$. For the entire profile $3-22 \mathrm{~km}$.

\section{References}

Gil, J.A. 1991, A\&A, 243, 219

Gould, D.M., \& Lyne, A.G. 1998, MNRAS, 301, 235

Kardashev N.S. et al. 1986, A\&A, 163, 114

Nowakowski, L.A. 1999a, "Fiducial Points In Pulsar Profiles, Superdispersion, and Vertical Mapping of the Magnetospheres", Ap.J, accepted for publication

Rankin, J.M. 1990, ApJ, 352, 247

Lyne, A.G., \& Manchester R.N. 1988, MNRAS, 234, 477 